\title{
ZEROS OF THE SUCCESSIVE DERIVATIVES OF HADAMARD GAP SERIES
}

\author{
ROBERT M. GETHNER
}

\begin{abstract}
A complex number $z$ is in the final set of an analytic function $f$, as defined by Pólya, if every neighborhood of $z$ contains zeros of infinitely many $f^{(n)}$. If $f$ is a Hadamard gap series, then the part of the final set in the open disk of convergence is the origin along with a union of concentric circles.
\end{abstract}

\section{INTRODUCTION}

A complex number $z$ is in the final set $\Lambda(f)$ of an analytic function $f$ if every neighborhood of $z$ contains zeros of infinitely many $f^{(n)}$. Final sets of various functions have been determined by Pólya $[4,5]$ (who introduced the notion) and others (see [2] for references). A power series

$$
f(z)=\sum_{k=0}^{\infty} c_{k} z^{N_{k}},
$$

with $c_{k} \neq 0$ for all $k$, has Hadamard gaps if there exists $L>1$ such that

$$
N_{k+1} / N_{k}>L \text { for all } k \geq 0 \text {. }
$$

Theorem 1. Let $f$ be a function whose Maclaurin series has Hadamard gaps and (finite or infinite) radius of convergence $R$. Then $\Lambda(f) \cap\{|z|<R\}=$ $\{0\} \cup\{z:|z| \in E\}$, where $E$ is closed in the topology of $(0, R)$.

Theorem 1 is best possible in the following sense.

Theorem 2. Let $R$ be in $(0, \infty]$, and let $E$ be closed in the topology of $(0, R)$. Then there exists a Hadamard gap series $f$ with radius of convergence $R$ such that $\Lambda(f) \cap\{|z|<R\}=\{0\} \cup\{|z|: z \in E\}$.

I am grateful to L. R. Sons and W. H. J. Fuchs for advice and encouragement.

\section{Proof of Theorem 1}

The proof of Theorem 1 depends on two lemmas, which I will prove in $\S \S 3$ and 4 , respectively, concerning functions $h$ of the form

$$
h(z)=\sum_{k=0}^{\infty} a_{k} z^{n_{k}} .
$$

Received by the editors November 16, 1990 and, in revised form, July 16, 1991.

1991 Mathematics Subject Classification. Primary 30D35, 30B10.

Key words and phrases. Final set, successive derivatives, Hadamard gaps, gap series. 
Fix such an $h$ and denote by $R$ the radius of convergence of the series. Set

$$
\mu(r)=\max \left\{\left|a_{k}\right| r^{n_{k}}: k \geq 0\right\}, \quad \nu(r)=\max \left\{k:\left|a_{k}\right| r^{n_{k}}=\mu(r)\right\} .
$$

(This notation is not standard; see [6, p. 3].) Finally, call a number $r$ in $(0, R)$ h-dominant if

$$
\sum_{k=0}^{\nu(r)-1}\left|a_{k}\right| r^{n_{k}}+\sum_{k=\nu(r)+1}^{\infty} n_{k}^{\nu(r)}\left|a_{k}\right| r^{n_{k}}<\mu(r),
$$

where the first sum is taken to be zero if $\nu(r)=0$.

The first lemma is an adaptation of [3, Theorem 6, p. 605]. Denote by $Z\left(s, t, \theta_{1}, \theta_{2}\right)$ the number of zeros (counting multiplicity) of $h$ in the set $\left\{r e^{i \theta}: s \leq r \leq t\right.$ and $\left.\theta_{1} \leq \theta \leq \theta_{2}\right\}$.

Lemma 1. Let $h$ have the form (2.1) (not necessarily with Hadamard gaps), and let $R, \mu(r)$, and $\nu(r)$ be as above. If $s$ and $t$ are $h$-dominant, if $s<t$, and if $0<\theta_{2}-\theta_{1}<2 \pi$, then

$$
\left|Z\left(s, t, \theta_{1}, \theta_{2}\right)-\left(n_{\nu(t)}-n_{\nu(s)}\right) \frac{\theta_{2}-\theta_{1}}{2 \pi}\right|<\nu(t)+2 .
$$

Lemma 2. Let $h$ and $R$ be as in Lemma 1, and suppose that there exists $L>1$ such that $n_{k+1} / n_{k}>L$ for all $k \geq 0$. Suppose also that

$$
n_{0} \geq \max \{9, \exp [\sqrt{(\log 6)(\log L)}]\} \text {. }
$$

Define

$$
\tau=54 e^{-2} /(\log L)(1-1 / L)\left(1-L^{-1 / 3}\right) .
$$

Then there is at least one h-dominant point in each interval $(C, D) \subset(0, R)$ such that

$$
\log (D / C)>\tau / n_{0}^{1 / 3} .
$$

Proof of Theorem 1. $0 \in \Lambda(f)$ by (1.2).

Define $h_{j}, a_{k}=a_{k}(j)$, and $n_{k}=n_{k}(j)$ by

$$
h_{j}(z)=z^{j} f^{(j)}(z)=\sum_{k=0}^{\infty} a_{k} z^{n_{k}}
$$

Then by (1.2),

$$
\text { (a) } n_{k+1} / n_{k}>L>1, \quad \text { (b) } n_{0} \geq j
$$

Define a set $E \subset(0, R)$ as follows: $r^{*} \in E$ if there exist an infinite set $T$ of positive integers and a sequence $\left\{r_{j}\right\}_{j \in T}$ such that

$$
\text { (a) } \lim _{j \rightarrow \infty, j \in T} r_{j}=r^{*}, \quad \text { (b) no } r_{j} \text { is } h_{j} \text {-dominant. }
$$

I will show that if $r^{*} \in E$ then $\left\{|z|=r^{*}\right\} \subset \Lambda(f)$, whereas if $r^{*} \notin E$ then $\left\{|z|=r^{*}\right\} \cap \Lambda(f)$ is empty.

Case I. $r^{*} \in E$. Choose $\left\{r_{j}\right\}$ as above and define $\tau$ by (2.5). By $(2.8 \mathrm{~b})$ and (2.9a), $r_{j} \exp \left\{2 \tau / n_{0}^{1 / 3}\right\}<R$ for all large $j$ in $T$. Pick such a $j>$ $\max \{9, \exp [\sqrt{(\log 6)(\log L)}]\}$. By Lemma 2, there are $h_{j}$-dominant points $s=$ $s(j)$ in $\left(r_{j} \exp \left\{-2 \tau / n_{0}^{1 / 3}\right\}, r_{j}\right)$ and $t=t(j)$ in $\left(r_{j}, r_{j} \exp \left\{2 \tau / n_{0}^{1 / 3}\right\}\right)$. 
Then

$$
\nu\left(s, h_{j}\right)<\nu\left(t, h_{j}\right) .
$$

For suppose that $\nu(s)=\nu(t) \equiv p$, and set

$$
\psi(r)=\frac{1}{\left|a_{p}\right| r^{n_{p}}}\left(\sum_{k=0}^{p-1}\left|a_{k}\right|^{r^{n_{k}}}+\sum_{k=p+1}^{\infty} n_{k}^{p}\left|a_{k}\right| r^{n_{k}}\right) .
$$

Then $\psi(s)<1$ and $\psi(t)<1$ by (2.3). Hence $\psi\left(r_{j}\right)<1$ since $\psi$ is convex [7, p. 172]. Thus $r_{j}$ is $h_{j}$-dominant, contrary to the definition of $r_{j}$. This proves (2.10).

Put

$$
U_{j}\left(\theta_{1}, \theta_{2}\right)=\left\{r e^{i \theta}: r_{j} \exp \left\{-2 \tau / n_{0}^{1 / 3}\right\} \leq r \leq r_{j} \exp \left\{2 \tau / n_{0}^{1 / 3} \text { and } \theta_{1} \leq \theta \leq \theta_{2}\right\}\right. \text {. }
$$

I will show that, if $j$ is sufficiently large, then

$h_{j}$ has at least one zero in $U_{j}\left(\theta_{1}, \theta_{2}\right)$ whenever $\theta_{2}-\theta_{1}>6 \pi L^{-j} /\left(1-L^{-1}\right)$.

For $x L^{-x} \downarrow$ for large $x$, so that, when $j \leq k,(2.8)$ and (2.10) give $k / n_{k}<$ $k L^{-k} / j \leq L^{-j}$ and $\left(n_{\nu(t)}-n_{\nu(s)}\right) / n_{\nu(t)}>1-L^{-[\nu(t)-\nu(s)]} \geq 1-L^{-1}$; thus, by Lemma 1 , the number of zeros in $U_{j}\left(\theta_{1}, \theta_{2}\right)$ is at least

$$
\begin{aligned}
Z\left(s, t, \theta_{1}, \theta_{2}\right) & \geq\left(n_{\nu(t)}-n_{\nu(s)}\right) \frac{\theta_{2}-\theta_{1}}{2 \pi}-\nu(t)-2 \\
& >\left(1-L^{-1}\right) n_{\nu(t)} \frac{\theta_{2}-\theta_{1}}{2 \pi}-3 \nu(t) \\
& >3 n_{\nu(t)} L^{-j}-3 \nu(t)>3 \nu(t)-3 \nu(t)=0,
\end{aligned}
$$

which establishes (2.11).

Now by (2.9a) and (2.8b), $r_{j} \exp \left\{-2 \tau / n_{0}^{1 / 3}\right\} \rightarrow r^{*}$ and $r_{j} \exp \left\{2 \tau / n_{0}^{1 / 3}\right\} \rightarrow r^{*}$ as $j \rightarrow \infty$ in $T$. Thus (2.11) implies that every point of $\left\{|z|=r^{*}\right\}$ is a limit point of zeros of $\left\{h_{j}\right\}_{j \in T}$, so that, by (2.7), $\left\{|z|=r^{*}\right\} \subset \Lambda(f)$.

Case II. $r^{*} \notin E$. For all large $j$ and small $\varepsilon, r$ is $h_{j}$-dominant for $r$ in $I \equiv\left(r^{*}-\varepsilon, r^{*}+\varepsilon\right)$. So by $(2.3)$,

$$
\left|h_{j}(z)\right| \geq \mu\left(r, h_{j}\right)-\sum_{k=0}^{\nu\left(r, h_{j}\right)-1}\left|a_{k}\right| r^{n_{k}}-\sum_{k=\nu\left(r, h_{j}\right)+1}^{\infty}\left|a_{k}\right| r^{n_{k}}>0
$$

whenever $|z|=r \in I$. This completes the proof of Theorem 1 .

\section{Proof of Lemma 1}

We need two more lemmas. The first is a variation on [6, Problem 66, p. 45]; the second is an adaptation of [3, Lemma 7]. Let $D$ denote differentiation.

Lemma 3. Let $J \equiv(a, b) \subset \mathbb{R}^{+}$, let $g: J \rightarrow \mathbb{C}$ be differentiable, and let $\alpha \in \mathbb{Z}^{+}$. If $\operatorname{Im}\{g\}$ changes sign at least twice in $J$, then $\operatorname{Im}\{(r D-\alpha) g\}$ changes sign there at least once.

Proof. For real $r$,

$$
\operatorname{Im}\{(r D-\alpha) g(r)\}=\operatorname{Im}\left\{r^{\alpha+1} \frac{d}{d r}\left[r^{-\alpha} g(r)\right]\right\}=r^{\alpha+1} \frac{d}{d r}\left[r^{-\alpha} \operatorname{Im}\{g(r)\}\right],
$$

and the lemma follows from Rolle's Theorem. 
For a function $H$ analytic on a contour $C$, denote by $\Delta(H, C)$ the variation over $C$ of any continuous branch of $\arg H$.

Lemma 4. Let $h$ have the form (2.1) with radius of convergence $R$, let $[s, t] \subset$ $(0, R)$, and suppose that $t$ is h-dominant. Set $I=I(\theta)=\left\{r e^{i \theta}: s \leq r \leq t\right\}$. If $h \neq 0$ on $I$, then $|\Delta(h, I)| \leq \pi[\nu(t)+1]$.

Proof. We may assume that $\theta=0$. Set $q=\nu(t)$. Choose $\phi$ so that $e^{i \phi} a_{q}$ is positive imaginary, and put

$$
H(r)=e^{i \phi}\left(r D-n_{0}\right)\left(r D-n_{1}\right) \cdots\left(r D-n_{q-1}\right) h(r) \equiv \sum_{k=n_{q}}^{\infty} b_{k} r^{n_{k}},
$$

where

$$
b_{k}=\left(n_{k}-n_{0}\right)\left(n_{k}-n_{1}\right) \cdots\left(n_{k}-n_{q-1}\right) e^{i \phi} a_{k} .
$$

I claim that $\operatorname{Im}\{H\}$ does not change sign in $(s, t)$. If the claim is correct, then $q$ applications of Lemma 3 show that $\operatorname{Im}\{h\}$ changes sign at most $q$ times in $(s, t)$, so that the curve $h(I)$ crosses the real axis at most $q$ times. Therefore $|\Delta(h, I)| \leq \pi(q+1)$, and the lemma follows.

To prove the claim, pick $r$ in $(s, t)$ and set

$$
\psi(r)=\frac{1}{\left|b_{q}\right| r^{n_{q}}}\left(\sum_{k=n_{q+1}}^{\infty}\left|b_{k}\right| r^{n_{k}}\right) .
$$

We have $\left|b_{k}\right| \leq n_{k}^{q}\left|a_{k}\right|$ and $\left|b_{q}\right| \geq\left|a_{q}\right|$ by (3.2). Thus, since $t$ is $h$-dominant and $q=\nu(t),(2.3)$ gives

$$
\psi(t) \leq \frac{1}{\left|a_{q}\right| t^{n_{q}}}\left(\sum_{k=n_{q+1}}^{\infty} n_{k}^{q}\left|a_{k}\right| t^{n_{k}}\right)=\frac{1}{\mu(t)}\left(\sum_{k=n_{\nu(t)+1}}^{\infty} n_{k}^{\nu(t)}\left|a_{k}\right| t^{n_{k}}\right)<1 .
$$

Now $\psi$ increases, so, by (3.1),

$$
\left|H(r)-b_{q} r^{n_{q}}\right| \leq \sum_{k=q+1}^{\infty}\left|b_{k}\right| r^{n_{k}}=\psi(r)\left|b_{q}\right| r^{n_{q}}<\left|b_{q}\right| r^{n_{q}} .
$$

But our choice of $\phi$ makes $b_{q} r^{n_{q}}$ positive imaginary, so that $H(r)$ is in the upper half-plane. This establishes the claim and Lemma 4.

Proof of Lemma 1. Let $\Gamma=I_{1} \cup C_{t} \cup I_{2} \cup C_{s}$, where $I_{1}=\left\{r e^{i \theta_{1}}: s \leq r \leq t\right\}, I_{2}=$ $\left\{r e^{i \theta_{2}}: s \leq r \leq t\right\}, C_{s}=\left\{s e^{i \theta}: \theta_{1} \leq \theta \leq \theta_{2}\right\}$, and $C_{t}=\left\{t e^{i \theta}: \theta_{1} \leq \theta \leq \theta_{2}\right\}$. Also put $P(z)=a_{\nu(s)} z^{n_{\nu(s)}}$ and $Q(z)=a_{\nu(t)} z^{n_{\nu(t)}}$.

First assume that $h \neq 0$ on $\Gamma$. Then

$$
\begin{aligned}
& \Delta(h, \Gamma)-\Delta\left(P, C_{s}\right)-\Delta\left(Q, C_{t}\right) \\
& \quad=\Delta\left(h / P, C_{s}\right)+\Delta\left(h / Q, C_{t}\right)+\Delta\left(h, I_{1}\right)+\Delta\left(h, I_{2}\right) .
\end{aligned}
$$

Also, (2.3) gives $|h(z) / P(z)-1|<1$, and hence $\operatorname{Re}\{h(z) / P(z)\}>0$, for $z \in C_{s}$. So $\left|\Delta\left(h / P, C_{s}\right)\right| \leq \pi$. Similarly, $\left|\Delta\left(h / Q, C_{t}\right)\right| \leq \pi$. Thus, by (3.3) and Lemma 4 ,

$$
\left|\Delta(h, \Gamma)-\left(n_{\nu(t)}-n_{\nu(s)}\right)\left(\theta_{2}-\theta_{1}\right)\right| \leq 2 \pi+2 \pi[\nu(t)+1],
$$


and Lemma 1 follows from the argument principle. If $h$ has zeros on $\Gamma$, apply (3.4) to a nearby contour $\Gamma^{\prime}$ on which $h \neq 0$ and let $\Gamma^{\prime} \rightarrow \Gamma$.

\section{Proof of Lemma 2}

Lemma 5. Let $h, R$, and $L$ be as in Lemma 2, and let (2.4) hold. Pick $m \geq 0$ and $[A, B] \subset(0, R)$, and suppose that

$$
\left|a_{k}\right| s^{n_{k}} \leq\left|a_{m}\right| s^{n_{m}} \quad \text { for all } k \geq 0 \text { and } s \text { in }[A, B]
$$

and

$$
\log \frac{B}{A}>\frac{6}{(\log L)(1-1 / L)} \frac{\left(\log n_{m}\right)^{2}}{n_{m}} .
$$

Then there exists $r$ in $(A, B)$ such that (2.3) holds with $\nu(r)=m$.

Proof of Lemma 2. For each $m \geq 0$, set $I_{m}=\left\{r \geq 0:\left|a_{m}\right| r^{n_{m}}=\mu(r, h)\right\}$. Denote by $(A, B)$ the interior of $I_{m} \cap(C, D)$. If $(A, B)$ has no $h$-dominant points, then (4.2) must fail. Therefore, since $\bigcup I_{m}=\mathbb{R}^{+}$,

$$
\begin{aligned}
\log \frac{D}{C} & =\int_{C}^{D} \frac{d x}{x}=\sum_{m=0}^{\infty} \int_{I_{m} \cap(C, D)} \frac{d x}{x} \\
& \leq \frac{6}{(\log L)(1-1 / L)} \sum_{m=0}^{\infty} \frac{\left(\log n_{m}\right)^{2}}{n_{m}} .
\end{aligned}
$$

Now $(\log x) / x^{1 / 3} \leq 3 / e$ for $x>0$; also, $n_{m}^{1 / 3}>\left(L^{m} n_{0}\right)^{1 / 3}$ by $(2.8 \mathrm{a})$. Thus $\left(\log n_{m}\right)^{2} / n_{m} \leq 9 e^{-2} n_{m}^{2 / 3} / n_{m}<9 e^{-2}\left(L^{m} n_{0}\right)^{-1 / 3}$. So by (4.3) and (2.5),

$$
\log \frac{D}{C} \leq \frac{1}{n_{0}^{1 / 3}} \frac{6}{(\log L)(1-1 / L)} \frac{9}{e^{2}} \sum_{m=0}^{\infty} L^{-m / 3}=\frac{\tau}{n_{0}^{1 / 3}}
$$

But this contradicts (2.6), and the proof is complete.

Proof of Lemma 5. Set

$$
\sigma=\exp \left\{\left(\log n_{m}\right)^{2} /\left(n_{m} \log L\right)\right\}
$$

Then

$$
\begin{gathered}
\sigma>1, \\
n_{k}^{m} \leq \sigma^{n_{k}} \text { for all } k \geq m, \\
\text { (a) } 2 \sigma^{n_{m}}\left\{\sigma(A / B)^{1 / 2}\right\}^{(1-1 / L) n_{m}}<\frac{1}{3}, \quad \text { (b) } \sigma(A / B)^{1 / 2}<1 .
\end{gathered}
$$

Proof of (4.6). By (2.8a) and (2.4), $k<\left(\log n_{k}\right) /(\log L)$. Also, $(\log x)^{2} / x$ decreases for $x>e^{2}$. Hence, by (4.4),

$$
\begin{aligned}
m \log n_{k} & \leq k \log n_{k} \leq \frac{\left(\log n_{k}\right)^{2}}{\log L}=\frac{\left(\log n_{k}\right)^{2}}{n_{k}} \frac{n_{k}}{\log L} \\
& \leq \frac{\left(\log n_{m}\right)^{2}}{n_{m}} \frac{n_{k}}{\log L}=n_{k} \log \sigma .
\end{aligned}
$$


Proof of (4.7). By (4.2) and (4.4), $(A / B)^{(1-1 / L) n_{m} / 2}<\sigma^{-3 n_{m}}$. By (2.4), $\sigma^{n_{m}} \geq$ $\sigma^{n_{0}} \geq 6$. Therefore

$$
\left\{\sigma(A / B)^{1 / 2}\right\}^{(1-1 / L) n_{m}}<\sigma^{(1-1 / L) n_{m}-3 n_{m}}<\sigma^{-n_{m}} \sigma^{-n_{m}} \leq \sigma^{-n_{m}} / 6 .
$$

This yields (4.7a), and (b) follows from (a) and (4.5).

We are now ready to prove $(2.3)$ with $\nu(r)=m$ and

$$
r=(A B)^{1 / 2} \text {. }
$$

When $k \geq m+1,(2.8 \mathrm{a})$ implies that

$$
n_{k}-n_{m}=\sum_{\gamma=m+1}^{k}\left(n_{\gamma}-n_{\gamma-1}\right) \geq \sum_{\gamma=m+1}^{k}(L-1) n_{\gamma-1} \geq(L-1) n_{m}(k-m) \text {. }
$$

By (4.6), (4.1) with $s=B$, and (4.8), and by (4.9), (which we may apply because of $(4.7 \mathrm{~b}))$,

$$
\begin{aligned}
n_{k}^{m}\left|a_{k}\right| r^{n_{k}} & \leq\left|a_{m}\right| B^{n_{m}}\left\{\sigma(A / B)^{1 / 2}\right\}^{n_{k}} \\
& \leq\left|a_{m}\right| r^{n_{m}} \sigma^{n_{m}}\left\{\sigma(A / B)^{1 / 2}\right\}^{(L-1)} n_{m}(k-m) .
\end{aligned}
$$

Next, if $0 \leq k<m-1$, then (2.8a) gives

$$
n_{k} \leq n_{m-1}=n_{m}-\left(n_{m}-n_{m-1}\right)<n_{m}-(1-1 / L) n_{m} \text {. }
$$

Thus, by (4.1) with $s=A$ and (4.8),

$$
\left.\left|a_{k}\right| r^{n_{k}} \leq\left|a_{m}\right| A^{n_{m}}\{(B / A)\}^{1 / 2}\right\}^{n_{k}} \leq\left|a_{m}\right| r^{n_{m}}\left\{(A / B)^{1 / 2}\right\}^{(1-1 / L) n_{m}} \text {. }
$$

We have $m \leq n_{m} \leq \sigma^{n_{m}}$ from (2.4) and (4.6). Also, $L-1>1-1 / L$ by (2.8a).

Thus (4.11), (4.10), (4.5), and (4.7a) give

$$
\begin{aligned}
& \sum_{k=0}^{m-1}\left|a_{k}\right| r^{n_{k}}+\sum_{k=m+1}^{\infty} n_{k}^{m}\left|a_{k}\right| r^{n_{k}} \\
& \quad<\left|a_{m}\right| r^{n_{m}}\left\{m\left[\sigma\left(\frac{A}{B}\right)^{1 / 2}\right]^{(1-1 / L) n_{m}}+\sigma^{n_{m}} \frac{\left[\sigma(A / B)^{1 / 2}\right]^{(1-1 / L) n_{m}}}{1-\left[\sigma(A / B)^{1 / 2}\right]^{(1-1 / L) n_{m}}}\right\} \\
& \leq\left|a_{m}\right| r^{n_{m}}\left\{\frac{\left[2 \sigma^{n_{m}}\right]\left[\sigma(A / B)^{1 / 2}\right]^{(1-1 / L) n_{m}}}{1-\left[\sigma(A / B)^{1 / 2}\right]^{(1-1 / L) n_{m}}}\right\}<\left|a_{m}\right| r^{n_{m}} \frac{1 / 3}{1-1 / 6}<\left|a_{m}\right| r^{n_{m}}
\end{aligned}
$$

This yields (2.3) and completes the proof of Lemma 5.

\section{Proof of Theorem 2}

The following construction is similar to that in [1].

Proof of Theorem 2. Pick positive sequences $\left\{r_{P}\right\}_{P=0}^{\infty},\left\{R_{P}\right\}_{P=0}^{\infty}$, and $\left\{\varepsilon_{P}\right\}_{P=0}^{\infty}$ so that

(a) the set of limit points of $\left\{r_{P}\right\}$ in $(0, R)$ is $E$,

(b) $r_{P} e^{\ell_{P}}<R_{P}<R$,

(c) $\varepsilon_{P} \rightarrow 0$,

(d) $R_{P} \rightarrow R$.

Choose a function $\psi: \mathbb{Z}^{+} \rightarrow \mathbb{R}^{+}$such that

$$
[\psi(x)]^{1 / x} \downarrow 1 / R \text { as } x \rightarrow \infty .
$$
Set

Define $f$ by (1.1), where $\left\{c_{k}\right\}$ and $\left\{N_{k}\right\}$ are defined inductively as follows.
(a) $N_{0}=3$,
(b) $c_{-1}=1$. 
Having chosen $c_{-1}, \ldots, c_{2 P-1}>0$ and $N_{0}, \ldots, N_{2 P}$, pick $c_{2 P}, c_{2 P+1}, N_{2 P+1}$, and $N_{2 P+2}$ as follows. Using (5.2) and (5.1b), pick $N_{2 P+1}$ large enough so that
(a) $N_{2 P+1} / N_{2 P}>2$,
(b) $N_{2 P+1}^{-N_{2 P}}>\left(e^{-\varepsilon_{P} / 2}\right)^{N_{2 P+1}-N_{2 P}}$,

and

$$
N_{2 P+1} \log \left\{\left[\psi\left(N_{2 P+1}\right)\right]^{1 / N_{2 P+1}} R_{P}\right\}<-2 \log 3,
$$

Set

$$
\text { (a) } c_{2 P+1}=\psi\left(N_{2 P+1}\right)
$$

(b) $c_{2 P}=c_{2 P+1} r_{P}^{N_{2 P+1}-N_{2 P}}$.

Finally, pick $N_{2 P+2}$ large enough so that
(a) $N_{2 P+2} / N_{2 P+1}>2$,
(b) $2 N_{2 P+1}^{2} \frac{\log N_{2 P+2}}{N_{2 P+2}}<\log 3$.

The function $f$ just constructed satisfies

$$
c_{2 P+1}^{1 / N_{2 P+1}} \geq c_{k}^{1 / N_{k}} \quad \text { for all } P \geq 0 \text { and } k \geq 2 P+1 .
$$

For odd $k,(5.9)$ follows from (5.7a) and (5.2). If $k \equiv 2 Q$ is even, (5.9) follows from (5.7) and (5.6) (both with $P$ replaced by $Q$ ):

$$
c_{2 Q}^{1 / N_{2 Q}}=\left(c_{2 Q+1}^{1 / N_{2 Q+1}} r_{Q}\right)^{N_{2 Q+1} / N_{2 Q}} r_{Q}^{-1}<c_{2 Q-1}^{1 / N_{2 Q-1}} \leq c_{2 P+1}^{1 / N_{2 P+1}}
$$

$f$ has radius of convergence $R$ by (5.7a), (5.2), and (5.9). By (1.1) and (5.8a), $0 \in \Lambda(f)$. We need the following lemma, proved at the end of the paper, to show that $\Lambda(f) \cap\{0<|z|<R\}=\{z:|z| \in E\}$.

Lemma 6. Let $f$ be as above, and define $\phi$ by

$$
f^{(j)}(z)=\sum_{N_{k} \geq j} c_{k} N_{k}\left(N_{k}-1\right) \cdots\left(N_{k}-j+1\right) z^{N_{k}-j} \equiv \sum_{N_{k} \geq j} \phi_{j k}(z) .
$$

If $P \geq 0$, and if

(a) $j \leq N_{2 P+1}$ and (b) $|z| \leq R_{P}$,

then

$$
\sum_{k=2 P+2}^{\infty}\left|\phi_{j k}(z)\right| \leq\left|\phi_{j, 2 P+1}(z)\right| / 2
$$

Proof that $\Lambda(f) \cap\{0<|z|<R\} \subset\{z:|z| \in E\}$. By (5.1), it is enough to show that $f^{(j)}(z) \neq 0$ whenever either

$$
j \in\left(N_{2 P}, N_{2 P+1}\right] \text { and } 0<|z| \leq R_{P}
$$

or

(5.14) $j \in\left(N_{2 P-1}, N_{2 P}\right]$ and $z \in\left\{0<|z| \leq r_{P} e^{-\varepsilon_{P}}\right\} \cup\left\{r_{P} e^{\varepsilon_{P}} \leq|z| \leq R_{P}\right\}$.

But if (5.13) holds, then (5.10) and (5.12) give

$$
\left|f^{(j)}(z)\right| \geq\left|\phi_{j, 2 P+1}(z)\right|-\sum_{k=2 P+2}^{\infty}\left|\phi_{j k}(z)\right| \geq\left|\phi_{j, 2 P+1}(z)\right| / 2>0 .
$$


If (5.14) holds, define

$$
G(j, P)=\frac{N_{2 P}\left(N_{2 P}-1\right) \cdots\left(N_{2 P}-j+1\right)}{N_{2 P+1}\left(N_{2 P+1}-1\right) \cdots\left(N_{2 P+1}-j+1\right)} .
$$

Then $G(j, P)>1 / N_{2 P+1}^{j}>N_{2 P+1}^{-N_{2 P}}$, so that, by $(5.4 \mathrm{~b})$,

$$
\left(e^{-\varepsilon_{P} / 2}\right)^{N_{2 P+1}-N_{2 P}}<G(j, P)<1 .
$$

Also, by (5.10), (5.15), and (5.7b),

$$
\left|\frac{\phi_{j, 2 P}(z)}{\phi_{j, 2 P+1}(z)}\right|=\frac{c_{2 P}}{c_{2 P+1}} G(j, P)|z|^{N_{2 P}-N_{2 P+1}}=G(j, P)\left(\frac{r_{P}}{|z|}\right)^{N_{2 P+1}-N_{2 P}} .
$$

If $r_{P} e^{\varepsilon_{P}} \leq|z| \leq R_{P}$, then $\left|\phi_{j, 2 P}(z) / \phi_{j, 2 P+1}(z)\right|<\left(e^{-\varepsilon_{P}}\right)^{N_{2 P+1}-N_{2 P}} \leq 3^{-6}$ by (5.17), (5.16), (5.4b), and (5.3). Hence, by (5.10) and (5.12),

$$
\begin{aligned}
\left|f^{(j)}(z)\right| & \geq \phi_{j, 2 P+1}(z)\left|-\sum_{k=2 P+2}^{\infty}\right| \phi_{j k}(z)|-| \phi_{j, 2 P}(z) \mid \\
& \geq\left|\phi_{j, 2 P+1}(z)\right|-\left|\phi_{j, 2 P+1}(z)\right| / 2-\left|\phi_{j, 2 P+1}(z)\right| / 3^{6}>0 .
\end{aligned}
$$

Similarly, if $0<|z| \leq r_{P} e^{-\varepsilon_{P}}$, then

$$
\left|\phi_{j, 2 P}(z) / \phi_{j, 2 P+1}(z)\right|>\left(e^{\varepsilon_{P} / 2}\right)^{N_{2 P+1}-N_{2 P}} \geq 3^{3}
$$

and

$$
\begin{aligned}
\left|f^{(j)}(z)\right| & \geq\left|\phi_{j, 2 P}(z)\right|-\sum_{k=2 P+2}^{\infty}\left|\phi_{j k}(z)\right|-\left|\phi_{j, 2 P+1}(z)\right| \\
& \geq\left|\phi_{j, 2 P}(z)\right|-\left(\frac{3}{2} \frac{1}{3^{3}}\right)\left|\phi_{j, 2 P+1}(z)\right|>0 .
\end{aligned}
$$

Proof that $\{z:|z| \in E\} \subset \Lambda(f)$. Fix $P$ and set

$$
r=[G(2 P, P)]^{1 /\left(N_{2 P+1}-N_{2 P}\right)} r_{P}
$$

Then, by (5.16),

$$
r \in\left(r_{P} e^{-\varepsilon_{P}}, r_{P}\right)
$$

Set $h_{j}(z)=z^{j} f^{(j)}(z)$. For $|z|=r$, we have

$$
\left|\phi_{2 P, 2 P}(z)\right|=\left|\phi_{2 P, 2 P+1}(z)\right|>\sum_{k=2 P+2}^{\infty}\left|\phi_{2 P, k}(z)\right|
$$

by (5.18), (5.17), and (5.12). Thus, by (5.10) and (2.2),

$$
\mu\left(r, h_{2 P}\right)=\left|z^{2 P} \phi_{2 P, 2 P}(z)\right|=\left|z^{2 P} \phi_{2 P, 2 P+1}(z)\right| \text {. }
$$

Therefore $r$ violates the definition (2.3) of $h_{2 P}$-dominance. It now follows from (5.19) and (5.1ca) that $E$ is in the set of limit points of the points which are not $h_{2 p}$-dominant. Thus $\{z:|z| \in E\} \subset \Lambda(f)$ by the paragraph containing (2.9). This completes the proof of Theorem 2 .

Proof of Lemma 6. Pick $k \geq 2 P+2$. By (5.9) and (5.7a),

$$
\begin{aligned}
\log \frac{c_{k}}{c_{2 P+1}} & =\frac{\log c_{k}}{N_{k}} N_{k}-\frac{\log c_{2 P+1}}{N_{2 P+1}} N_{2 P+1} \\
& \leq\left(N_{k}-N_{2 P+1}\right) \frac{\log \psi\left(N_{2 P+1}\right)}{N_{2 P+1}} .
\end{aligned}
$$


Also, $(\log x) / x$ decreases for $x>N_{2 P+1}$ by (5.3a). Thus, by (5.8),

$$
\begin{aligned}
N_{2 P+1} \frac{\log N_{k}}{N_{k}-N_{2 P+1}} & =\frac{N_{2 P+1}}{1-N_{2 P+1} / N_{k}} \frac{\log N_{k}}{N_{k}} \\
& <\frac{N_{2 P+1}}{1 / 2} \frac{\log N_{2 P+2}}{N_{2 P+2}} \leq \frac{\log 3}{N_{2 P+1}} .
\end{aligned}
$$

By (5.10), (5.11), (5.20), (5.21), and (5.5),

$$
\begin{aligned}
\log \left|\frac{\phi_{j k}(z)}{\phi_{j, 2 P+1}(z)}\right| & \leq \log \frac{c_{k}}{c_{2 P+1}}+N_{2 P+1} \log N_{k}+\left(N_{k}-N_{2 P+1}\right) \log R_{P} \\
& \leq\left(N_{k}-N_{2 P+1}\right)\left[\log \left(\left\{\psi\left(N_{2 P+1}\right)\right\}^{1 / N_{2 P+1}} R_{P}\right)+\frac{\log 3}{N_{2 P+1}}\right] \\
& <\left(N_{k}-N_{2 P+1}\right)\left(\frac{-\log 3}{N_{2 P+1}}\right) .
\end{aligned}
$$

But $\left(N_{k}-N_{2 P+1}\right) / N_{2 P+1} \geq k-2 P-1$ by (5.4a), (5.8a), and (4.9) (with $L=2$, $N_{k}$ in place of $n_{k}$, and $\left.m=2 P+1\right)$. Thus (5.22) gives

$$
\sum_{k=2 P+2}^{\infty}\left|\frac{\phi_{j k}(z)}{\phi_{j, 2 P+1}(z)}\right| \leq \sum_{k=2 P+2}^{\infty} e^{-(\log 3)(k-2 P-1)}=\frac{1 / 3}{1-1 / 3}=\frac{1}{2} .
$$

\section{REFERENCES}

1. A. Edrei and G. R. Maclane, On the zeroes of the derivatives of an entire function, Proc. Amer. Math. Soc. 8 (1957), 702-706.

2. R. M. Gethner, Zeros of the successive derivatives of Hadamard gap series in the unit disk, Michigan Math. J. 36 (1989), 403-414.

3. W. K. Hayman, Angular value distribution of power series with gaps, Proc. London Math. Soc. (3) 24 (1972), 590-624.

4. G. Pólya, Über die Nullstellen sukzessiver Derivierten, Collected Papers (R. P. Boas, ed.), MIT Press, Cambridge, Mass., 1974.

5. - On the zeros of the derivatives of a function and its analytic character, Collected Papers (R. P. Boas, ed.), MIT Press, Cambridge, Mass., 1974.

6. G. Pólya and G. Szegö, Problems and theorems in analysis, vol. 2, Springer-Verlag, New York, 1972.

7. E. C. Titchmarsh, The theory of functions, 2nd ed., Oxford Univ. Press, Oxford, 1939.

Department of Mathematics, Franklin and Marshall College, Lancaster, PennsylVANIA 17604-3003

E-mail address: rgethner@acad.fandm.edu 